Proceedings of the 45th International School and Conference on the Physics of Semiconductors "Jaszowiec" 2016, Szczyrk

\title{
The Effect of Substrate on Vibrational Properties of Single-Layer $\mathrm{MoS}_{2}$
}

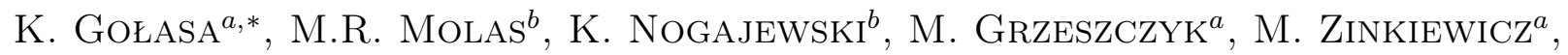 \\ M. POTEMSKI ${ }^{b}$ AND A. BABIŃSKI ${ }^{a}$ \\ ${ }^{a}$ Faculty of Physics, University of Warsaw, L. Pasteura 5, 02-093 Warsaw, Poland \\ ${ }^{b}$ Laboratoire National des Champs Magnétiques Intenses, CNRS-UGA-UPS-INSA-EMFL, \\ 25 rue des Martyrs, 38042 Grenoble, France
}

\begin{abstract}
We report on the Raman scattering from single-layer molybdenum disulfide $\left(\mathrm{MoS}_{2}\right)$ deposited on various substrates: $\mathrm{Si} / \mathrm{SiO}_{2}$, hexagonal boron nitride (h-BN), sapphire, as well as suspended. Room temperature Raman scattering spectra are investigated under both resonant $(632.8 \mathrm{~nm})$ and non-resonant $(514.5 \mathrm{~nm})$ excitations. A rather weak influence of the substrate on the Raman scattering signal is observed. The most pronounced, although still small, is the effect of h-BN, which manifests itself in the change of energy positions of the $E^{\prime}$ and $A_{1}^{\prime}$ Raman modes of single-layer $\mathrm{MoS}_{2}$. We interpret this modification as originating from van der Waals interaction between the $\mathrm{MoS}_{2}$ and h-BN layers.
\end{abstract}

DOI: $10.12693 /$ APhysPolA.130.1172

PACS/topics: 63.22.--m, 71.35.--y, 78.20.-e, 78.30.--j

\section{Introduction}

Fast progress in graphene research during last decade has recently triggered off intensive investigations of other two-dimensional (2D) materials [1-3]. In particular, much attention of the worldwide scientific community has been attracted by layered transition metal dichalcogenides (TMDs) of a general formula $\mathrm{MX}_{2}$, where $\mathrm{M}$ stands for a group VI transition metal atom like Mo or W and $\mathrm{X}=(\mathrm{S}, \mathrm{Se}$ or $\mathrm{Te})$ is a chalcogen atom [4]. Similarly to graphite, the atoms within individual $X-M-X$ planes of TMDs crystals are bound one to another by strong ion-covalent bonds, whereas weak out-of-plane van der Waals interaction holds the adjacent $X-M-X$ layers together. Thanks to this property, TMDs can readily be thinned down to the single-layer thickness via mechanical exfoliation or chemical treatment. Such a process significantly affects their electronic structure and optical properties leading, for instance, to the indirect-to-direct band gap crossover in the case of semiconducting TMDs. This makes 2D counterparts of bulk TMDs crystals very promising candidates for potential applications in optoelectronics, sensors, catalysis or spintronics $[2,5-7]$. Molybdenum disulfide $\left(\mathrm{MoS}_{2}\right)$ is the best known representative of the TMDs family [1]. It has been suggested that in the monolayer $(1 \mathrm{ML})$ form it could complement graphene in optoelectronic applications because contrary to graphene $1 \mathrm{ML} \mathrm{MoS}_{2}$ is a semiconductor with a nonzero direct energy band gap equal to $1.88 \mathrm{eV}$. This makes the investigation of basic properties of $1 \mathrm{ML} \mathrm{MoS}$ of importance for development of novel semiconductor devices.

In this paper we present a study of the effect of substrate on the crystal lattice dynamics in monolayer

*corresponding author; e-mail: katarzyna.golasa@fuw.edu.pl
$\mathrm{MoS}_{2}$ deposited on various substrates: standard $\mathrm{Si} / \mathrm{SiO}_{2}$, $\mathrm{Si} / \mathrm{SiO}_{2}$ with perforated $\mathrm{SiO}_{2}$ layer (free-standing $1 \mathrm{ML}$ $\mathrm{MoS}_{2}$ ), hexagonal boron nitride (h-BN), and sapphire. Our technique of choice is the Raman spectroscopy which constitutes a non-destructive and effective method for probing the electronic and vibrational properties of different types of materials. In the case of TMDs it is widely used to determine the number of layers and the amount of strain in flakes under consideration $[8,9]$. The main features of the non-resonantly excited Raman spectrum of bulk $\mathrm{MoS}_{2}$ are four modes: $E_{2 \mathrm{~g}}^{2}, E_{1 \mathrm{~g}}, E_{2 \mathrm{~g}}^{1}$, and $A_{1 \mathrm{~g}}[10]$. The frequency difference between the latter two modes allows to establish the thickness of few-layer $\mathrm{MoS}_{2}$ flakes [8]. In $1 \mathrm{ML} \mathrm{MoS}_{2}$, the Raman-active modes $E_{2 \mathrm{~g}}^{1}$ and $A_{1 \mathrm{~g}}$ transform into the $E^{\prime}$ and $A_{1}^{\prime}$ vibrations, respectively.

\section{Samples and experiment}

Samples studied in this work were prepared by means of mechanical exfoliation of bulk crystals purchased from Nanoscience Instruments, Inc. $\left(\mathrm{MoS}_{2}\right)$ and HQ Graphene (h-BN). The flakes were first exfoliated onto a polydimethylsiloxane (PDMS) stamp attached to a glass plate. Monolayer flakes of $\mathrm{MoS}_{2}$ were then identified by their optical contrast and cross-checked by the Raman scattering and photoluminescence (PL) measurements. In order to deposit them on target substrates $\left(\mathrm{Si} /(320 \mathrm{~nm}) \mathrm{SiO}_{2}\right.$ with and without perforated $\mathrm{SiO}_{2}$ layer and sapphire) an all-dry PDMS-based transfer method similar to the one described in Ref. [11] was employed. Prior to transferring, all substrates had been cleaned in acetone, isopropanol, de-ionized water and ashed with oxygen plasma. $\mathrm{MoS}_{2} / \mathrm{h}$-BN heterostructures were prepared by means of two subsequent flake transfers: first a few-layer flake of h-BN was deposited on a $\mathrm{Si} /(320 \mathrm{~nm}) \mathrm{SiO}_{2}$ substrate and then a $1 \mathrm{ML}$ 
$\mathrm{MoS}_{2}$ was put on its top. Perforation of the $\mathrm{SiO}_{2}$ layer with holes of $10 \mu \mathrm{m}$ diameter was done with the use of reactive-ion etching in trifluoromethane and a lithographically-defined hard aluminium mask which was then removed from the $\mathrm{Si} / \mathrm{SiO}_{2}$ substrate by dissolving in a base solution.

The Raman scattering experiments were carried out at room temperature $(300 \mathrm{~K})$, under a resonant $(632.8 \mathrm{~nm}$, $1.96 \mathrm{eV})$ and non-resonant $(514.5 \mathrm{~nm}, 2.41 \mathrm{eV})$ excitation, provided by a continuous wave $\mathrm{He}-\mathrm{Ne}$ and $\mathrm{Ar}^{+}$laser, respectively. The excitation light was focused by means of a $50 \times$ long working distance objective. The signal was collected via the same microscope objective, sent through an $0.5 \mathrm{~m}$ monochromator equipped with 300 , 900 , and 1800 grooves $/ \mathrm{mm}$ gratings and detected by a charge-coupled-device camera. The laser spot diameter on the sample was equal to $\approx 1 \mu \mathrm{m}$ and the laser power was kept at the level of $150 \mu \mathrm{W}$ in order to avoid heating effects.

\section{Results and discussion}

Optical microscope images of the $1 \mathrm{ML} \mathrm{MoS}_{2}$ flakes deposited on non-perforated and perforated $\mathrm{Si} / \mathrm{SiO}_{2}$, $\mathrm{Si} / \mathrm{SiO}_{2} / \mathrm{h}-\mathrm{BN}$, and sapphire are presented in Fig. 1.
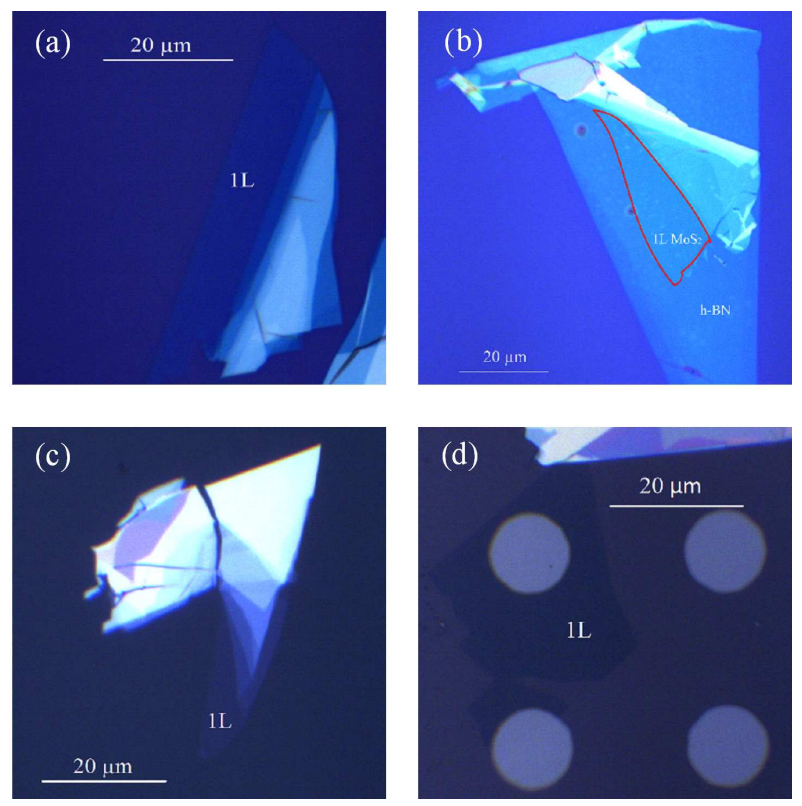

Fig. 1. Optical microscope images of $1 \mathrm{ML} \mathrm{MoS}_{2}$ flakes deposited onto (a) $\mathrm{Si} / \mathrm{SiO}_{2}$, (b) $\mathrm{Si} / \mathrm{SiO}_{2} / \mathrm{h}-\mathrm{BN}$, (c) sapphire and (d) $\mathrm{Si} / \mathrm{SiO}_{2}$ with $10 \mu \mathrm{m}$ holes etched into the $\mathrm{SiO}_{2}$ layer (free-standing case).

The Raman spectra measured on $1 \mathrm{ML} \mathrm{MoS}_{2}$ deposited on various substrates $\left(\mathrm{Si} / \mathrm{SiO}_{2}, \mathrm{~h}-\mathrm{BN}\right.$, and sapphire) as well as suspended are shown in Fig. 2. As can be seen, the intensities of the $E^{\prime}$ and $A_{1}^{\prime}$ modes are substantially affected by the substrate (the changes cover more than one order of magnitude). The highest intensities of these modes are observed for the flake placed on the $\mathrm{Si} / \mathrm{SiO}_{2}$ substrate, while the smallest ones were measured for the suspended flake. A similar effect has already been reported for monolayer $\mathrm{MoS}_{2}$ deposited on $\mathrm{SiO}_{2}$ and h$\mathrm{BN}[12]$. The frequencies and characteristic widths of the observed modes are also slightly modified by the substrate. In order to quantify their changes, we fitted the experimental data (solid circles) with a combination of two Lorentzian curves (solid lines). All fitting parameters (the central frequency, the full width at half maximum (FWHM), and the intensity of the spectral line) are presented for both the $E^{\prime}$ and $A_{1}^{\prime}$ modes in Fig. 3.

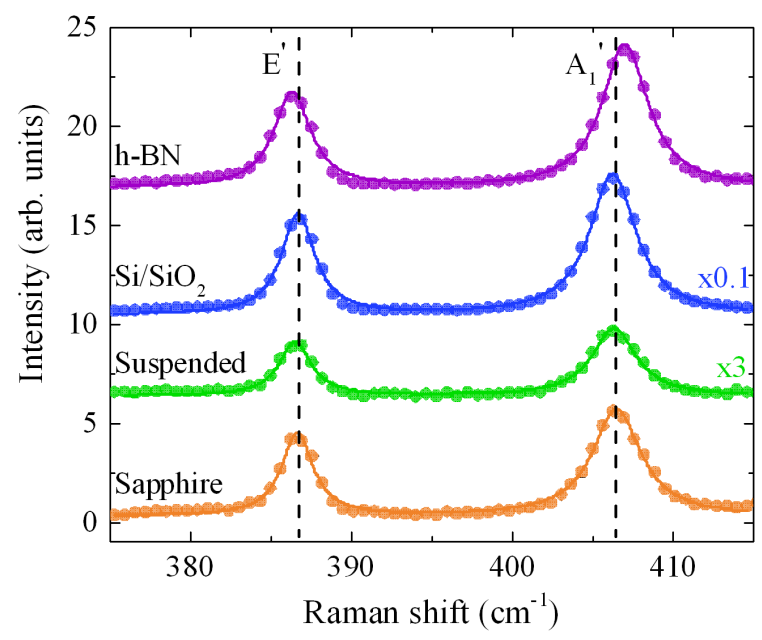

Fig. 2. Room-temperature Raman scattering spectra of $1 \mathrm{ML} \mathrm{MoS}_{2}$ deposited on $\mathrm{Si} / \mathrm{SiO}_{2}$, h-BN, and sapphire, as well as suspended, measured with $2.41 \mathrm{eV}$ $(514.5 \mathrm{~nm})$ excitation. Solid circles are the experimental data and continuous curves are Lorentzian fits to the Raman-active $E^{\prime}$ and $A_{1}^{\prime}$ modes.
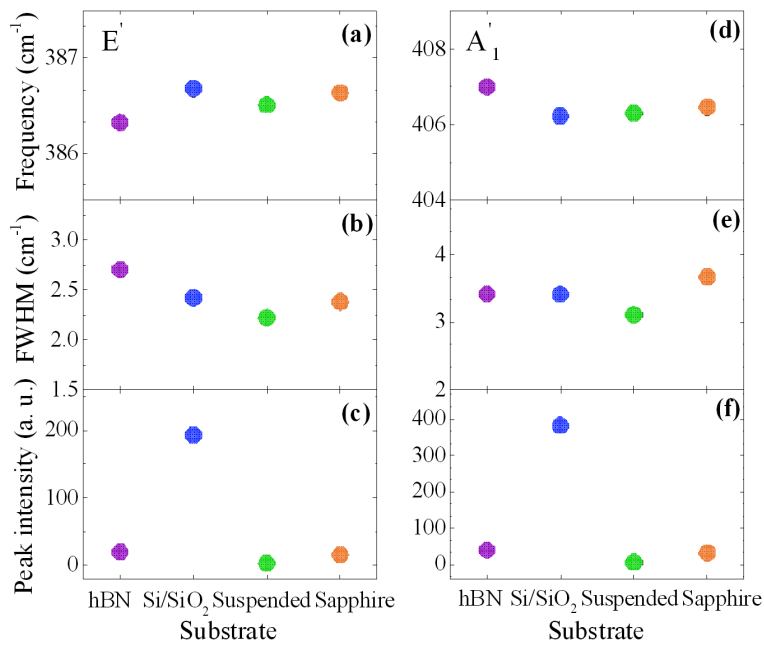

Fig. 3. Frequency, FWHM and peak intensity of the $E^{\prime}$ (left) and $A_{1}^{\prime}$ (right) Raman-active modes in $1 \mathrm{ML}$ $\mathrm{MoS}_{2}$ deposited on various substrates.

The maximum frequency shift (the distance between the two outer frequency positions) amounts to $\approx 0.364 \mathrm{~cm}^{-1}$ and $\approx 0.77 \mathrm{~cm}^{-1}$ for the $E^{\prime}$ and $A_{1}^{\prime}$ modes, 
respectively. The highest frequency of the $E^{\prime}$ mode is observed for $1 \mathrm{ML} \mathrm{MoS}$ deposited on $\mathrm{Si} / \mathrm{SiO}_{2}$ substrate, while the lowest one for the flake transferred on h-BN. The opposite behaviour is seen for the $A_{1}^{\prime}$ peak. An increase in the FWHM of the $E^{\prime}$ mode on h-BN and of the $A_{1}^{\prime}$ mode on sapphire can also be noticed. Moreover, a prominent increase (by a factor of $\approx 5$ ) in the Raman intensity of both the $E^{\prime}$ and $A_{1}^{\prime}$ modes on $\mathrm{Si} / \mathrm{SiO}_{2}$ is observed. In our opinion, the effect of substrate material on the intensity of the Raman peaks under analysis may be related to the interference (constructive or destructive) between the laser light and the measured signal.

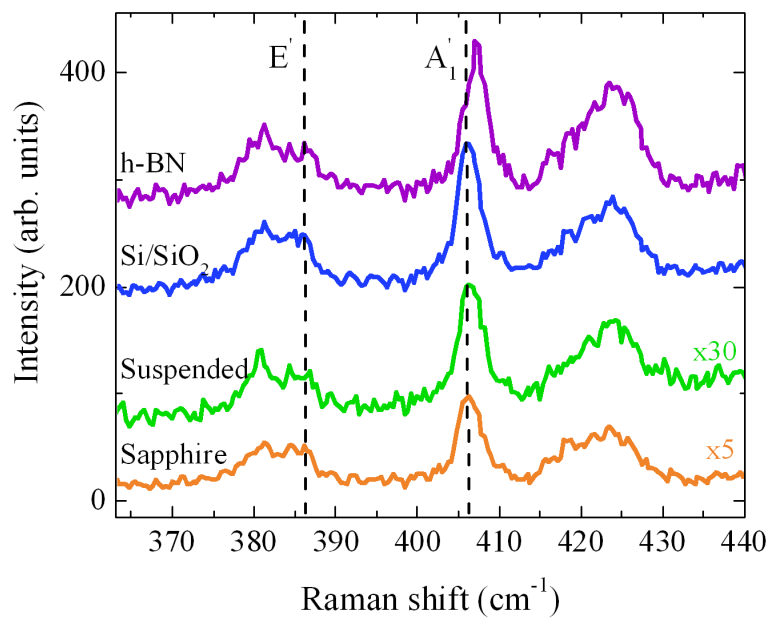

Fig. 4. Room-temperature Raman scattering spectra of $1 \mathrm{ML} \mathrm{MoS}$ flakes deposited on various substrates $\left(\mathrm{Si} / \mathrm{SiO}_{2}, \mathrm{~h}-\mathrm{BN}\right.$, and sapphire) and suspended, measured with $1.96 \mathrm{eV}(632.8 \mathrm{~nm})$ excitation.

Resonantly excited Raman spectra of $1 \mathrm{ML} \mathrm{MoS}_{2}$ deposited on various substrates and suspended are shown in Fig. 4. While the non-resonant Raman spectrum is dominated by the two aforementioned modes due to zonecenter first-order Raman scattering [13], the resonant spectrum consists of more spectral lines [14]. The scattering processes probed under resonant excitation are enhanced by an interaction of phonons with excitonic electron transitions $[14,15]$. In this excitation regime, the effect of substrate on the $A_{1}^{\prime}$ mode's intensity is different from the one observed for the non-resonant excitation (compare Fig. 2 and Fig. 4). Identical to the non-resonant excitation conditions it seems that the frequency positions of the $E^{\prime}$ and $A_{1}^{\prime}$ modes (see Fig. 4) do not significantly change with the substrate. Only the frequency of the $A_{1}^{\prime}$ mode in $1 \mathrm{ML} \mathrm{MoS}_{2}$ deposited on h-BN shows a sizeable blue shift of about $1.07 \mathrm{~cm}^{-1}$. Moreover, despite the noise in the experimental data, a double structure of this mode can be noticed. This may be related to different interaction of the monolayer $\mathrm{MoS}_{2}$ flake with the substrate in different regions of the excited sample.

Most interesting is the observed blue shift of the $A_{1}^{\prime}$ mode's energy accompanied by a smaller red shift of the
$E^{\prime}$ mode's energy. It is well known that interlayer interactions in multiple-layer TMDs result in a strong (weak) dependence of the $A_{1 \mathrm{~g}} / A_{1}^{\prime}\left(E_{2 \mathrm{~g}}^{1} / E^{\prime}\right)$ mode's energy on the sample thickness [16, 17]. A similar effect can be expected for heterostructures composed of flakes of different materials as in both cases the individual layers are held together by van der Waals interactions. We believe that this explains the influence of thin-film h-BN substrate on the Raman scattering spectrum of $1 \mathrm{ML} \mathrm{MoS}_{2}$ we see.

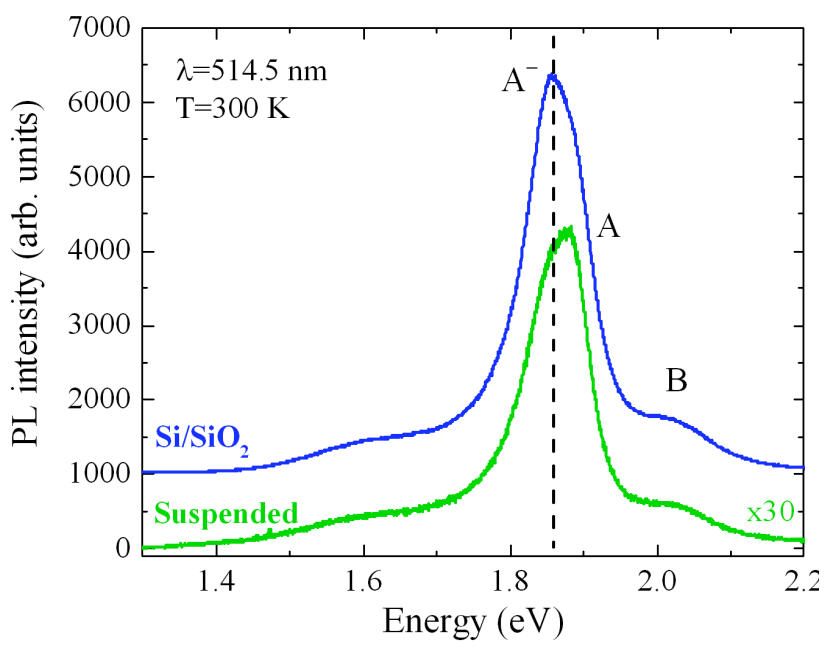

Fig. 5. Room-temperature PL spectra of suspended (green line) and $\mathrm{Si} / \mathrm{SiO}_{2}$ supported (blue line) $1 \mathrm{ML}$ of $\mathrm{MoS}_{2}$ measured under non-resonant $(514.5 \mathrm{~nm})$ excitation.

In addition to the Raman scattering experiments described above, we also measured the PL spectra of $1 \mathrm{ML}$ $\mathrm{MoS}_{2}$ flakes deposited on $\mathrm{Si} / \mathrm{SiO}_{2}$ substrate and suspended (see Fig. 5). As indicated in the figure, in the high energy range they comprise two lines labelled $\mathrm{A}$ and $\mathrm{B}$, which appear at $\approx 1.88 \mathrm{eV}$ and $\approx 2.03 \mathrm{eV}$, respectively. They originate from the creation of two possible neutral excitons at the $K$ point of the Brillouin zone that are associated with two spin-orbit split subbands in the valence band [1]. Moreover, another feature labelled $A^{-}$ can be seen at $\approx 1.86 \mathrm{eV}$. We ascribe it to the charged exciton (trion) of the $A$ transition [12]. As clearly visible, its relative intensity with respect to the $A$ line varies with dielectric properties of the flake's environment. Finally, it is worth pointing out that the intensity of PL from the suspended flake is significantly weaker (around 30 times) in comparison with the intensity of PL from the flake deposited on $\mathrm{Si} / \mathrm{SiO}_{2}$. This remains in agreement with the change of the Raman modes intensity under the same excitation conditions.

\section{Conclusions}

In conclusion, we have studied the optical properties of $1 \mathrm{ML} \mathrm{MoS}$ flakes deposited on various substrates as well as suspended. We measured their room-temperature 
Raman scattering spectra under both the resonant and non-resonant excitation. We found that the optical properties of $1 \mathrm{ML} \mathrm{MoS}$ may be slightly affected by the substrate. It particularly concerns the intensities and energy positions of the $E^{\prime}$ and $A_{1}^{\prime}$ Raman-active modes. The most pronounced effect is observed for the h-BN substrate. The blue shift of the $A_{1}^{\prime}$ mode's energy in the $1 \mathrm{ML} \mathrm{MoS}_{2} / \mathrm{h}-\mathrm{BN}$ heterostructure can be attributed to van der Waals interactions between the few-layer h-BN and $1 \mathrm{ML} \mathrm{MoS}$ flakes.

\section{Acknowledgments}

This work has been supported by the National Science Centre under grants no. DEC-2013/11/N/ST3/04067 and DEC-2015/16/T/ST3/00496. Funding from European Graphene Flagship and European Research Council (ERC-2012-AdG-320590-MOMB) is also acknowledged.

\section{References}

[1] K.F. Mak, C. Lee, J. Hone, J. Shan, T.F. Heinz, Phys. Rev. Lett. 105, 136805 (2010).

[2] A. Splendiani, L. Sun, Y. Zhang, T. Li, J. Kim, C.-Y. Chim, G. Galli, F. Wang, Nano Lett. 10, 1271 (2010).

[3] Q.H. Wang, K. Kalantar-Zadeh, A. Kis, J.N. Coleman, M.S. Strano, Nature Nanotech. 7, 699 (2012).

[4] X. Huang, Z. Zeng, H. Zhang, Chem. Soc. Rev. 42, 1934 (2013).

[5] Z. Yin, Hai Li, Hong Li, L. Jiang, Y. Shi, Y. Sun, G. Lu, Q. Zhang, X. Chen, H. Zhang, ACS Nano 6, 74 (2012).
[6] A. Castellanos-Gomez, M. Barkelid, A.M. Goossens, V.E. Calado, H.S.J. van der Zant, G.A. Steele, Nano Lett. 12, 3187 (2012).

[7] Hai Li, Z. Yin, Q. He, Hong Li, X. Huang, G. Lu, D.W. Fam, A.I. Tok, Q. Zhang, H. Zhang, Small 8, 63 (2012).

[8] C. Lee, H. Yan, L.E. Brus, T.F. Heinz, J. Hone, S. Ryu, ACS Nano 4, 2695 (2010).

[9] C. Rice, R.J. Young, R. Zan, U. Bangert, D. Wolverson, T. Georgiou, R. Jalil, K.S. Novoselov, Phys. Rev. B 87, 081307 (2013).

[10] C.V. Ramana, U. Becker, V. Shutthanandan, C.M. Julien, Geochem. Trans. 9, 8 (2008).

[11] A. Castellanos-Gomez, M. Buscema, R. Molenaar, V. Singh, L. Janssen, H.S.J. van der Zant, G.A. Steele, 2D Mater. 1, 011002 (2014).

[12] M. Buscema, G.A. Steele, H.S.J. van der Zant, A. Castellanos-Gomez, Nano Res. 7, 561 (2015).

[13] T.J. Wieting, J.L. Verbie, Phys. Rev. B 3, 4286 (1971).

[14] K. Gołasa, M. Grzeszczyk, P. Leszczyński, C. Faugeras, A.A.L. Nicolet, A. Wysmołek, M. Potemski, A. Babiński, Appl. Phys. Lett. 104, 092106 (2014).

[15] A.M. Stacy, D.T. Hodul, J. Phys. Chem. Solids 46 , 405 (1985).

[16] M. Grzeszczyk, K. Gołasa, M. Zinkiewicz, K. Nogajewski, M.R. Molas, M. Potemski, A. Wysmołek, A. Babiński, 2D Mater. 3, 2 (2016).

[17] G. Froehlicher, E. Lorchat, F. Fernique, C. Joshi, A. Molina-Sánchez, L. Wirtz, S. Berciaud, Nano Lett. 15, 6481 (2015). 